\title{
Classical Fano oscillator
}

\author{
D. M. Riffe \\ Department of Physics, Utah State University, Logan, Utah 84322-4415, USA
}

(Received 3 May 2011; revised manuscript received 20 July 2011; published 31 August 2011)

\begin{abstract}
Starting from the quantum-mechanical Fano-Anderson Hamiltonian, we derive classical equations of motion for coordinates associated with the discrete and continuum states. The frequency-dependent absorption spectrum associated with this classical system exhibits the same Fano line shape as the quantum-mechanical system when appropriate correspondences between classical and quantum variables are made. In the time domain, the response of this classical Fano oscillator depends upon the asymmetry parameter $q$ that appears in the expression for the Fano line shape. In particular, under the influence of impulsive driving of the system, the discrete oscillator's phase changes by $\pm \pi / 2$ as $q$ increases from zero (maximum asymmetry in the frequency domain) to $\mp \infty$ (minimum asymmetry). Previously published ultrafast-laser-pulse-driven coherent-phonon oscillations in degenerate $p$-type Si [K. Kato et al., Jpn. J. Appl. Phys. 48, 100205 (2009)] are discussed in light of these theoretical results.
\end{abstract}

PACS number(s): 63.20.K-, 78.47.jg

\section{INTRODUCTION}

In 1961, Fano published a (now classic) theoretical treatment of the interaction of a discrete quantum state with a background of continuum of states under the condition that both the discrete and the continuum levels are excited by some external perturbation. ${ }^{1}$ The major result of that paper is an asymmetric resonant line shape (known as the Fano profile) associated with absorption by the coupled system. The line shape is characterized by a frequency shift $\Delta \Omega_{0}$ and width parameter $\Gamma$ that depend upon the coupling of the discrete excitation with the continuum and an asymmetry parameter $q$ that depends upon the coupling as well as the relative excitation strengths associated with the discrete and continuum levels. Subsequent to Fano's paper, this asymmetric resonance has been observed in a wide variety of solid-state settings, including ultraviolet absorption by excitons in insulators, ${ }^{2}$ infrared absorption by adsorbates on metal surfaces, ${ }^{3-5}$ and Raman scattering of optical vibrations in semiconductors. ${ }^{6-8}$ The Fano resonance is also a key element of the optics of plasmonic nanostructures and metamaterials. ${ }^{9}$

Raman scattering in degenerate $p$-type $\mathrm{Si}$ exhibits one of the cleanest examples of a Fano system. In $p$-type $\mathrm{Si}$, the discrete excitation-the optic phonon-couples to the continuum of electronic excitations associated with intervalence-band transitions. Raman spectra obtained over a wide range of incident photon energies and doping levels are well fit by the Fano line shape, ${ }^{6-8}$ and the line-shape parameters are quantitatively described by detailed theory. ${ }^{10,11}$ Local phonon modes associated with the boron impurity in $p$-type $\mathrm{Si}$ also exhibit the Fano line shape. ${ }^{12,13}$

As with Raman scattering in $\mathrm{Si}$, other Fano systems have been predominantly measured in the frequency domain. Recently, however, there have been several time-domain investigations (of $\mathrm{Zn}, \mathrm{Bi}$, and lightly doped $n$-type $\mathrm{Si}$ ) that have been interpreted in terms of the discrete-state-continuum-state physics that gives rise to Fano line shapes. ${ }^{14-17}$ In these systems, the discrete level is also a phonon oscillation (that is coherently excited by an ultrashort laser pulse), and the coupled background is electronic in nature (with perhaps some vibrational contribution in Bi). Quantitative information has been extracted from the data by fitting the Fourier transform (of the time-domain oscillations) with the Fano line shape. A slight twist to the standard Fano picture in these experiments is that the coupled continuum is modified by the exciting laser pulse; because of this, the line-shape asymmetry, width, and shift depend upon the laser-pulse intensity, with all three generally increasing as the intensity increases. ${ }^{16,17}$

Time-domain coherent-phonon data have also been obtained from degenerate $p$-type $\mathrm{Si}^{18}$ These data exhibit a curious feature that is directly observed in the time domain: The phase of the coherent oscillations in the heavily doped material is substantially different than the phase in lightly doped Si. This observation raises the following question: Is this phase difference due to the discrete-phonon-electroniccontinuum coupling that is responsible for the Fano line shape observed in Raman spectra from degenerate $p$-type $\mathrm{Si}$ ?

Motivated by these time-domain results, especially this most recent one from degenerate $p$-type $\mathrm{Si}$, and because coherent-phonon oscillations are a classical manifestation of the interaction of a femtosecond light pulse with a solid, we have studied classical equations of motion associated with the coordinates of a quantum-mechanical Fano model. We first demonstrate that the classical system exhibits the same frequency-dependent Fano profile as the quantum-mechanical system. We then consider the time-domain response of the system. In particular, we find that, when driven by impulsive forcing (as is the case for the coherent phonon in the experiments on heavily doped $p$-type $\mathrm{Si}$ ), the phase of the induced oscillations associated with the discrete oscillator is a simple function of the Fano asymmetry parameter $q$. For large $|q|$ (which corresponds to weak excitation of the continuum), the oscillations exhibit the expected sinelike behavior. However, as $q$ approaches zero, the oscillations continuously become more cosinelike. As we demonstrate, these results from our model are consistent with the phase difference observed in the $\mathrm{Si}$ experiments. ${ }^{18}$

\section{FANO-ANDERSON MODEL}

Due to its bilinear coupling of the discrete and continuum states, the simplest quantum-mechanical model that exhibits 
the Fano line shape is the Fano-Anderson Hamiltonian, ${ }^{19}$ which may be expressed as

$$
H=E_{0} \alpha^{\dagger} \alpha+\sum_{k} E_{k} b_{k}^{\dagger} b_{k}+\sum_{k} V_{k}\left(\alpha b_{k}^{\dagger}+\alpha^{\dagger} b_{k}\right),
$$

where $E_{0}$ is the excitation energy of the discrete state $|\alpha\rangle, E_{k}$ is the excitation energy associated with the continuum state $|k\rangle$, and $V_{k}$ (assumed to be real) is the matrix element that couples the discrete state to the $k$ th continuum excitation. The external perturbation is introduced through matrix elements $M_{\alpha g}=\langle\alpha|\mathbf{M}| g\rangle$ and $M_{k g}=\langle k|\mathbf{M}| g\rangle$ between the ground state of the system and the discrete and continuum excited states, respectively.

The absorption spectrum $I(\omega)$ associated with the external perturbation $\mathbf{M}$ is given by

$$
I(\omega)=2 \pi \omega \sum_{j}\left|M_{j g}\right|^{2} \delta\left(\hbar \omega-E_{j}^{\prime}\right),
$$

where $j$ labels an eigenstate of the Hamiltonian $H$ with energy $E_{j}^{\prime}$. As has been previously shown, ${ }^{1,5,20}$ the absorption spectrum has the Fano line shape,

$$
I(\omega)=I_{B}(\omega) \frac{(q+\epsilon)^{2}}{1+\epsilon^{2}},
$$

where

$$
I_{B}(\omega)=2 \pi \omega \sum_{k}\left|M_{k g}\right|^{2} \delta\left(\hbar \omega-E_{k}\right)
$$

is the absorption by the background continuum in the absence of coupling to the discrete state. The parameter $\epsilon=2\left(\omega-\Omega_{0}-\Delta \Omega_{0}\right) / \Gamma$ is the normalized frequency, where $\Omega_{0}=E_{0} / \hbar$,

$$
\Delta \Omega_{0}=\frac{1}{\hbar} \mathbb{P} \sum_{k} \frac{\left|V_{k}\right|^{2}}{\hbar \omega-E_{k}}
$$

and

$$
\Gamma=\frac{2 \pi}{\hbar} \sum_{k}\left|V_{k}\right|^{2} \delta\left(\hbar \omega-E_{k}\right) .
$$

Here, $\mathbb{P}$ indicates the principal value. The asymmetry parameter $q$ can be expressed as

$$
q=\frac{M_{g \alpha}+\mathbb{P} \sum_{k} M_{g k} V_{k} /\left(\hbar \omega-E_{k}\right)}{\pi \sum_{k} M_{g k} V_{k} \delta\left(\hbar \omega-E_{k}\right)} .
$$

Typically, the range of frequencies over which the coupling is manifest is quite narrow compared to $\Omega_{0}$. In this case, $\hbar \omega$ in Eqs. (5)-(7) can be replaced by $\hbar \Omega_{0}$, making $\Delta \Omega_{0}, \Gamma$, and $q$ all frequency independent. $1,5,20$

In Fig. 1, we plot $I(\omega) / I_{B}(\omega)$ vs $\epsilon$ for several values of $q$. Generally, the line shape is asymmetric with a dip into the background absorption that goes to zero at one particular frequency (when $\epsilon=-q$ ). There are two interesting limits to the line shape. The first limit is when the external perturbation $\mathbf{M}$ does not couple to the background states $|k\rangle$. In this case, $M_{g k} \rightarrow 0,|q| \rightarrow \infty$, and the line shape becomes a symmetric Lorentzian. The second limit is when $\mathbf{M}$ does not couple to the discrete state $|\alpha\rangle$. In this case, $M_{g \alpha}=0$, typically making $q$ very small (if not zero). In the limit $q \rightarrow 0$, the absorption

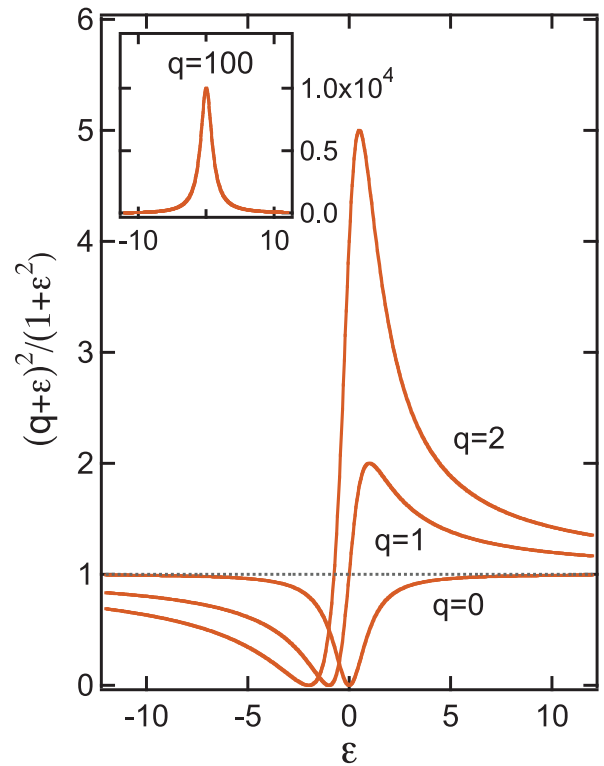

FIG. 1. (Color online) Fano line shape for several values of the asymmetry parameter $q$.

profile becomes a symmetric antiresonance in the continuum absorption as Fig. 1 illustrates.

\section{THE CLASSICAL FANO OSCILLATOR}

\section{A. Equations of motion}

We derive classical equations of motion for coordinates $Q=\left\langle\alpha+\alpha^{\dagger}\right\rangle$ and $q_{k}=\left\langle b_{k}+b_{k}^{\dagger}\right\rangle$, assuming bosonic commutation relations for the raising and lowering operators, e.g., $\left[\alpha, \alpha^{\dagger}\right]=1$. Using $i \hbar \frac{d\langle A\rangle}{d t}=\langle[A, H]\rangle$, it is straightforward to obtain the coupled equations of motion,

$$
\hbar^{2} \frac{d^{2} Q}{d t^{2}}+\left(E_{0}^{2}+\sum_{k} V_{k}^{2}\right) Q+\sum_{k} V_{k}\left(E_{0}+E_{k}\right) q_{k}=0,
$$

and

$$
\hbar^{2} \frac{d^{2} q_{k}}{d t^{2}}+E_{k}^{2} q_{k}+\sum_{k^{\prime} \neq k} V_{k^{\prime}}^{2} q_{k^{\prime}}+V_{k}\left(E_{0}+E_{k}\right) Q=0 .
$$

We now consider motion of coordinate $Q$ under the influence of an external driving force $F_{Q}(t)$ on the discrete oscillator and forces $F_{k}(t)$ on the background oscillators. We also ignore terms of order $V_{k}^{2}$ and define classical coupling coefficients $c_{k}=V_{k}\left(E_{0}+E_{k}\right) / \hbar^{2}$. Because there is no average absorption for harmonic driving forces without damping explicitly included, we include damping in each of the $q_{k}$ equations through a damping parameter $\gamma_{k}$, although for small enough damping, the results are independent of $\gamma_{k}$. With these modifications, the equations of motion can be written as

$$
\frac{d^{2} Q}{d t^{2}}+\Omega_{0}^{2} Q+\sum_{k} c_{k} q_{k}=F_{Q}(t)
$$

and

$$
\frac{d^{2} q_{k}}{d t^{2}}+\gamma_{k} \frac{d q_{k}}{d t}+\omega_{k}^{2} q_{k}+c_{k} Q=F_{k}(t)
$$


where $\omega_{k}=E_{k} / \hbar$. As we now show, Eqs. (10) and (11) result in a Fano profile that is equivalent to that of the quantummechanical system; we, thus, take Eqs. (10) and (11) as the defining equations for the classical Fano oscillator.

\section{B. Frequency-domain response}

The frequency-dependent response of the system is found by considering driving forces of the form $F_{i}(t)=\operatorname{Re}\left(\tilde{F}_{i} e^{-i \omega t}\right)$ and assuming that the coordinate time dependence can also be written as $Q(t)=\operatorname{Re}\left(\tilde{Q} e^{-i \omega t}\right)$ and $q_{k}(t)=\operatorname{Re}\left(\tilde{q}_{k} e^{-i \omega t}\right)$. For this case, Eqs. (10) and (11) become

$$
\left(\Omega_{0}^{2}-\omega^{2}\right) \tilde{Q}+\sum_{k} c_{k} \tilde{q}_{k}=\tilde{F}_{Q}
$$

and

$$
\left(\omega_{k}^{2}-\omega^{2}-i \gamma_{k} \omega\right) \tilde{q}_{k}+c_{k} \tilde{Q}=\tilde{F}_{k} .
$$

These equations can be straightforwardly solved for $\tilde{Q}$ and $\tilde{q}_{k}$ as

$$
\tilde{Q}=\frac{\tilde{F}_{Q}-\sum_{k} c_{k} \tilde{F}_{k} / Z_{k}}{Z_{Q}-\sum_{k} c_{k}^{2} / Z_{k}}
$$

and

$$
\tilde{q}_{k}=\frac{1}{Z_{k}}\left(\tilde{F}_{k}-c_{k} \frac{\tilde{F}_{Q}-\sum_{k} c_{k} \tilde{F}_{k} / Z_{k}}{Z_{Q}-\sum_{k} c_{k}^{2} / Z_{k}}\right),
$$

where $Z_{Q}=\Omega_{0}^{2}-\omega^{2}$ and $Z_{k}=\omega_{k}^{2}-\omega^{2}-i \gamma_{k} \omega$. Under the assumption that $\tilde{F}_{Q}$ and $\tilde{F}_{k}$ are real, the frequency-dependent absorption can be calculated via

$$
I(\omega)=\frac{\omega}{2} \operatorname{Im}\left(\sum_{k} \tilde{F}_{k} \tilde{q}_{k}+\tilde{F}_{Q} \tilde{Q}\right),
$$

which results in

$$
\begin{aligned}
I(\omega)= & \frac{\omega}{2} \operatorname{Im}\left[\sum_{k} \frac{\tilde{F}_{k}^{2}}{Z_{k}}+\frac{1}{Z_{Q}-\sum_{k} c_{k}^{2} / Z_{k}}\right. \\
& \left.\times\left(\tilde{F}_{Q}-\sum_{k} c_{k} \tilde{F}_{k} / Z_{k}\right)^{2}\right] .
\end{aligned}
$$

We note that the first term in Eq. (17) is the background absorption $I_{B}$. Generally, Eq. (17) has the Fano line shape with line-shape parameters given by

$$
\begin{aligned}
\Delta \Omega_{0} & =-\frac{1}{2 \omega} \operatorname{Re} \sum_{k} \frac{c_{k}^{2}}{Z_{k}}, \\
\Gamma & =\frac{1}{\omega} \operatorname{Im} \sum_{k} \frac{c_{k}^{2}}{Z_{k}},
\end{aligned}
$$

and

$$
q=\frac{\tilde{F}_{Q}-\Sigma^{\prime}}{\Sigma^{\prime \prime}}+\frac{2 I_{B} \Gamma-\Sigma^{\prime \prime 2}}{\left(\tilde{F}_{Q}-\Sigma^{\prime}\right) \Sigma^{\prime \prime}},
$$

where $\Sigma^{\prime}$ and $\Sigma^{\prime \prime}$ are the real and imaginary parts of $\sum_{k} c_{k} \tilde{F}_{k} / Z_{k}$.

With a few approximations, this classical Fano line shape essentially becomes identical to that of the quantum system. If we take the limit $\gamma_{k}=\gamma \rightarrow 0$, then, for positive frequencies $(w>0)$, expressions of the form $\sum_{k} A_{k} / Z_{k}$ can be expressed for good approximation as

$$
\begin{aligned}
\sum_{k} \frac{A_{k}}{Z_{k}}= & \frac{1}{2 \omega} \mathbb{P} \sum_{k} \frac{A_{k}}{\omega_{k}-\omega} \\
& +\frac{i \pi}{2 \omega} \sum_{k} A_{k} \delta\left(\omega-\omega_{k}\right),
\end{aligned}
$$

which allows us to write $\Delta \Omega_{0}$ and $\Gamma$ as

$$
\Delta \Omega_{0}=\mathbb{P} \sum_{k} \frac{\left(c_{k} / 2 \omega\right)^{2}}{\omega-\omega_{k}},
$$

and

$$
\Gamma=2 \pi \sum_{k}\left(c_{k} / 2 \omega\right)^{2} \delta\left(\omega-\omega_{k}\right) .
$$

If we further assume that the coupling coefficients $c_{k}$ and forces $F_{k}$ only depend upon $k$ via $\omega_{k}$, then the second term in Eq. (20) vanishes and $q$ reduces to

$$
q=\frac{\tilde{F}_{Q}+\mathbb{P} \sum_{k} \tilde{F}_{k}\left(c_{k} / 2 \omega\right) /\left(\omega-\omega_{k}\right)}{\pi \sum_{k} \tilde{F}_{k}\left(c_{k} / 2 \omega\right) \delta\left(\omega-\omega_{k}\right)} .
$$

Comparing Eqs. (22)-(24) with their quantum counterparts, Eqs. (5)-(7), we see that they are identical with the correspondences $\tilde{F}_{Q} / \tilde{F}_{k} \leftrightarrow M_{g \alpha} / M_{g k}$ and $c_{k} / 2 \omega \leftrightarrow V_{k} / \hbar$. This last correspondence is not quite equivalent to the definition $c_{k}=V_{k}\left(E_{0}+E_{k}\right) / \hbar^{2}$. However, relevant values of $\omega$ and $\omega_{k}$ are typically not too different from the discrete frequency $\Omega_{0}$. Thus, in Eqs. (22)-(24), $c_{k} / 2 \omega$ can be substituted with $c_{k} /\left(\Omega_{0}+\omega_{k}\right)=V_{k} / \hbar$ without appreciably altering their values.

We are aware of several other calculations of coupled oscillators in the context of Fano-like response. We emphasize, however, that none of these previously investigated models include a true continuum background (of multiple oscillators) coupled to the discrete oscillator. In an investigation of the interaction of an adsorbate vibrational mode with a continuum of excitations in the underlying solid, Sorbello considered the coupling of a single oscillator with either one (highly damped) background oscillator $\left(Z_{B}=\omega_{B}^{2}-\omega^{2}-i \omega \gamma_{B}\right)$ or a viscous bath $\left(Z_{B}=-i \omega \gamma_{B}\right) .^{5}$ Both of these systems exhibit the Fano line shape in the vicinity of the single oscillator's natural frequency. Indeed, our results, as expressed in Eqs. (18)-(20), reduce to those of Sorbello in the limit of one background oscillator and the replacement of $Z_{k}$ by either of Sorbello's $Z_{B}$ 's. Similarly, in their investigation of a sharp waveguide mode with a broad plasmon mode, Klein et al. also calculated the absorption associated with two coupled oscillators and pointed out that the response in the vicinity of the sharp mode appears Fano-like. ${ }^{21}$ Joe et al. investigated this same classical system in the context of understanding the interaction of a discrete quantum-dot electronic state with a continuum of waveguide states. ${ }^{22}$ We also note the coupled-oscillator modeling of infrared reflectivity spectra from the high-dielectricconstant materials $\mathrm{BaTiO}_{3}, \mathrm{SrTiO}_{3}$, and $\mathrm{KTaO}_{3}$ by Barker and Hopfield. ${ }^{23}$ Although Barker and Hopfield did not make the connection to Fano's paper, their elegant analysis was later extended to several oscillators by Struzhkin et al. as the basis for modeling Fano-like features in the infrared vibrational 
spectra of ice. ${ }^{24}$ There are also examples from plasmonicnanomaterial optics where several coupled oscillators are used to reproduce Fano-like spectra. ${ }^{25-27}$

\section{Time-domain response}

We now consider Eqs. (10) and (11) with more general forces. In particular, we consider these equations with forces that last for a finite time, and our goal is to find $Q(t)$ after the forces have stopped acting on the system. We start with the ansatz that the solutions can be written as a power series in the coupling coefficients $c_{k}$,

$$
Q(t)=Q^{(0)}(t)+\sum_{k} c_{k} Q_{k}^{(1)}(t)+\cdots,
$$

and

$$
q_{k}(t)=q_{k}^{(0)}(t)+c_{k} q_{k}^{(1)}(t)+\cdots,
$$

and that, for sufficiently weak coupling, each series can be truncated at the terms linear in the $c_{k}$ 's. [It can be shown, using the Laplace transform, that a sufficient condition for this truncation is $\sum_{k}\left(c_{k} / \omega_{k}\right)^{2} \ll \Omega_{0}^{2}$. This inequality is typically satisfied if $\Gamma \ll \Omega_{0}$, as can be ascertained from Eq. (23).] With this ansatz, the equations of motion simplify to a set of hierarchal equations, the first three of which are

$$
\begin{gathered}
\frac{d^{2} Q^{(0)}}{d t^{2}}+\Omega_{0}^{2} Q^{(0)}=F_{Q}(t), \\
\frac{d^{2} q_{k}^{(0)}}{d t^{2}}+\gamma_{k} \frac{d q_{k}^{(0)}}{d t}+\omega_{k}^{2} q_{k}^{(0)}=F_{k}(t),
\end{gathered}
$$

and

$$
\frac{d^{2} Q_{k}^{(1)}}{d t^{2}}+\Omega_{0}^{2} Q_{k}^{(1)}=-q_{k}^{(0)} .
$$

We further assume initial conditions $Q(t \rightarrow-\infty)=0$, $\dot{Q}(t \rightarrow-\infty)=0, q_{k}(t \rightarrow-\infty)=0$, and $\dot{q}_{k}(t \rightarrow-\infty)=0$. As outlined in Appendix A, the asymptotic solution for $Q(t)$ to linear order in the $c_{k}$ 's can be written as

$$
\begin{aligned}
Q(\bar{t})= & \sqrt{2 \pi} \operatorname{Im}\left[\left(\frac{\hat{F}_{0}\left(-\Omega_{0}\right)}{\Omega_{0}}+\sum_{k} \frac{c_{k}}{\Omega_{k}} \frac{\hat{F}_{k}\left(-\Omega_{0}\right)}{\Omega_{0}^{2}-\omega_{k}^{2}-i \gamma_{k} \Omega_{0}}\right)\right. \\
& \left.\times \exp \left(i \Omega_{0} \bar{t}\right)\right]
\end{aligned}
$$

where $\hat{F}_{i}(\Omega)$ is the Fourier transform of $F_{i}(t), \Omega_{k}=$ $\sqrt{\omega_{k}^{2}-\gamma_{k}^{2} / 4}$, and $\bar{t}$ indicates the long-time solution. For symmetric impulsive forces centered at $t=0, \hat{F}_{i}(-\Omega)$ is real and $\hat{F}_{i}(-\Omega)=\hat{F}_{i}(\Omega)$. In this case, Eq. (30) can be written as

$$
\begin{aligned}
Q(\bar{t})= & \frac{\sqrt{2 \pi}}{\Omega_{0}}\left[\left(\hat{F}_{Q}\left(\Omega_{0}\right)+\mathbb{P} \sum_{k} \frac{c_{k}}{2 \omega_{k}} \frac{\hat{F}_{k}\left(\Omega_{0}\right)}{\Omega_{0}-\omega_{k}}\right) \sin \left(\Omega_{0} \bar{t}\right)\right. \\
& \left.+\left(\pi \sum_{k} \frac{c_{k}}{2 \omega_{k}} \hat{F}_{k}\left(\Omega_{0}\right) \delta\left(\Omega_{0}-\omega_{k}\right)\right) \cos \left(\Omega_{0} \bar{t}\right)\right], \quad(31)
\end{aligned}
$$

in the limit that $\gamma_{k}=\gamma$ becomes negligibly small. [The evolution of $Q(t)$ toward this asymptotic limit is discussed in Appendix B.]

Comparing Eq. (24) with Eq. (31), we see that the ratio of the amplitudes that multiply the $\sin \left(\Omega_{0} \bar{t}\right)$ and $\cos \left(\Omega_{0} \bar{t}\right)$ terms in

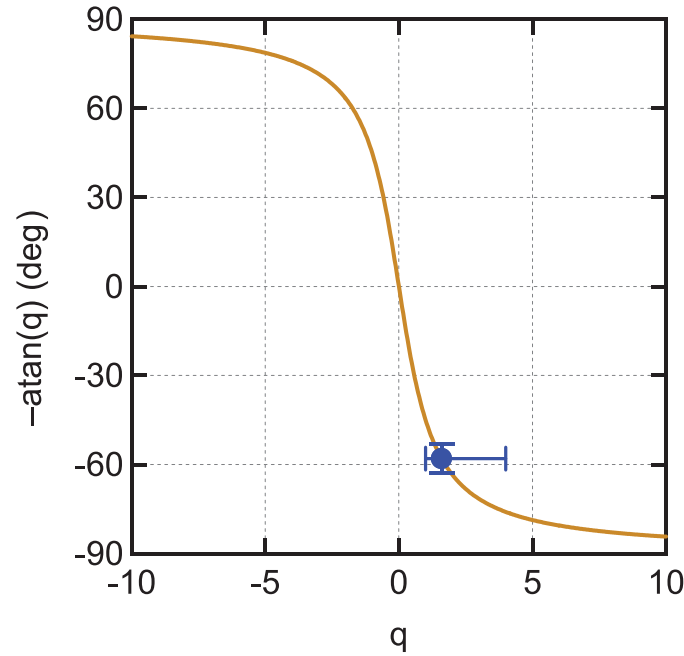

FIG. 2. (Color online) Phase $\phi=-\operatorname{atan}(q)$ vs $q$ for the classical Fano oscillator. The solid circle (with vertical error bars included) is the phase that has been measured for a degenerate $p$-type Si sample ( $p$ between $3 \times 10^{19}$ and $\left.1.5 \times 10^{20} \mathrm{~cm}^{-3}\right){ }^{18}$ the horizontal line spans the range of estimated $q$ values for the sample as deduced from Fig. 3 (see text for details).

Eq. (31) is essentially the classical Fano asymmetry parameter $q$ evaluated at $\omega=\Omega_{0}$. The only differences are as follows: (i) the appearance of the force Fourier transforms $\hat{F}_{i}\left(\Omega_{0}\right)$ rather than the harmonic force amplitudes $\tilde{F}_{i}$ and (ii) the presence of $1 / \omega_{k}$ rather than $1 / \omega$ in the sums over $k$, which is typically of little consequence. Thus, we can express Eq. (31) as

$$
\begin{aligned}
Q(t)= & \frac{\sqrt{2 \pi}}{\Omega_{0}}\left(\hat{F}_{Q}\left(\Omega_{0}\right)+\mathbb{P} \sum_{k} \frac{c_{k}}{2 \omega_{k}} \frac{\hat{F}_{k}\left(\Omega_{0}\right)}{\Omega_{0}-\omega_{k}}\right) \\
& \times\left[\sin \left(\Omega_{0} t\right)+\frac{1}{q} \cos \left(\Omega_{0} t\right)\right],
\end{aligned}
$$

or equivalently,

$$
\begin{aligned}
Q(t)= & \left(\hat{F}_{Q}\left(\Omega_{0}\right)+\mathbb{P} \sum_{k} \frac{c_{k}}{2 \omega_{k}} \frac{\hat{F}_{k}\left(\Omega_{0}\right)}{\Omega_{0}-\omega_{k}}\right) \\
& \times \sqrt{1+q^{-2}} \cos \left[\Omega_{0} t-\operatorname{atan}(q)\right] .
\end{aligned}
$$

In Fig. 2, we plot the phase $\phi=-\operatorname{atan}(q)$ as a function of the asymmetry parameter $q$. The extreme limits of $q$ on the behavior of $Q(t)$ are evident from Eqs. (32) and (33) and from the figure. If the background oscillators are not driven, $1 / q=0, \operatorname{atan}(q)= \pm \pi / 2,{ }^{28}$ and the oscillations are sinelike, as expected for impulsive driving of a single oscillator. On the other hand, if only the background oscillators are driven, then $q=0, \operatorname{atan}(q)=0$, and the oscillations are cosinelike.

\section{COHERENT PHONONS IN SILICON}

Raman-scattering spectra from heavily doped (degenerate) $p$-type Si exhibit a Fano line shape in the vicinity of the Si optic phonon. Here, the Fano line shape arises from the interaction of the optic phonon with a valence-band electronic continuum as both the phonon and the electronic continuum Raman scatter the incident radiation. In Fig. 3, we summarize the results of two studies in which $q$ has been determined as a function of 


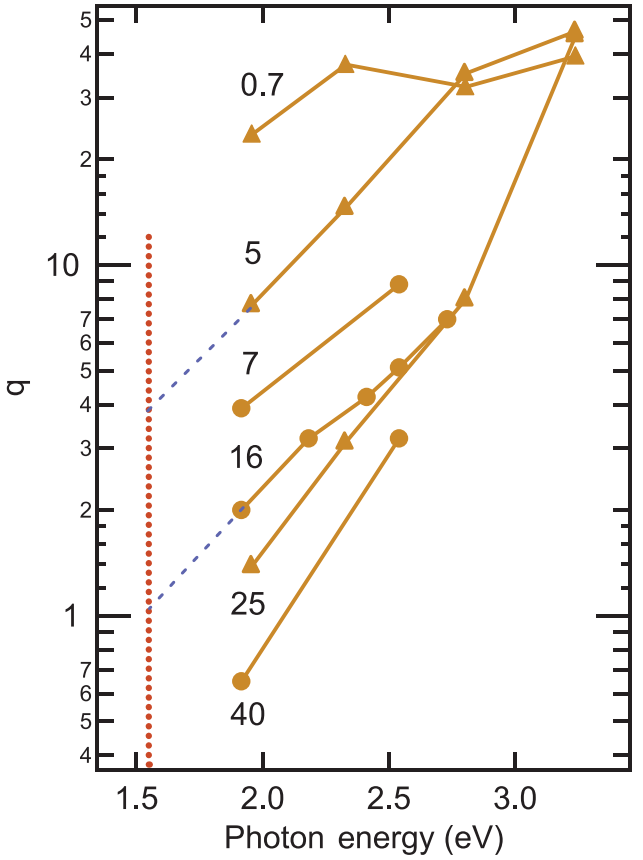

FIG. 3. (Color online) Experimental Fano asymmetry parameter $q$ deduced from Raman-scattering data vs photon energy $\hbar \omega$ for degenerate $p$-type $\mathrm{Si}$. Indicated doping density for each curve is in units of $10^{19} \mathrm{~cm}^{-3}$. Triangles are from Ref. 8, and circles are from Ref. 7. Dotted lines are extrapolations of the $5 \times 10^{19}$ and $1.6 \times$ $10^{20} \mathrm{~cm}^{-3}$ data to $\hbar \omega=1.55 \mathrm{eV}$.

photon energy for a total of six different doping densities. ${ }^{7,8}$ For photon energies away from the strong Raman-scattering resonance at $\sim 3.4 \mathrm{eV}$ (associated with direct valence-band-toconduction-band transitions), ${ }^{6,8,29}$ the data show the expected monatomic dependence of $q$ on doping density: more heavily doped samples exhibit smaller $q$ values, owing to stronger scattering by the continuum with increased doping.

Recently, Kato et al. measured the coherent response of the Si optic phonon in a degenerate $p$-type sample using ultrafast pump-probe reflectivity at $1.55-\mathrm{eV}$ photon energy. ${ }^{18}$ From their data, it can be deduced that the phase (as defined above) of the coherent oscillations in their degenerate sample is $32 \pm 5^{\circ}$ larger than in undoped $\mathrm{Si}$. The phase in undoped $\mathrm{Si}$ is $( \pm) 90^{\circ}$ (due to purely impulsive excitation of the optic phonon by transiently stimulated Raman scattering); ${ }^{30,31}$ therefore, the phase of the heavily doped sample in the paper by Kato et al. is $-58 \pm 5^{\circ}$ (when constrained within the bounds $-\pi / 2<\phi<\pi / 2){ }^{32}$

In light of our time-domain result for the classical Fano oscillator, the significant phase shift in the optic-phonon oscillations in degenerate $p$-type Si suggests that the difference in phase between undoped and heavily doped $\mathrm{Si}$ is due to the time-domain interaction between the concurrently excited discrete optic phonon and the valence-band continuum. A quantitative comparison supports this conjecture. As Ramanscattering measurements with $1.55-\mathrm{eV}$ photons on degenerate $p$-type Si have not been reported, we use Fig. 3 to estimate $q$ at $1.55 \mathrm{eV}$ for the Kato sample. The Kato sample has a reported value of $p$ between $3 \times 10^{19}$ and $1.5 \times 10^{20} \mathrm{~cm}^{-3} .{ }^{18}$ As several of the curves in Fig. 3 illustrate, vs photon energy $\log (q)$ varies linearly as the photon energy approaches $1.55 \mathrm{eV}$. Thus, we linearly extrapolate the $5 \times 10^{19}$ - and $1.6 \times 10^{20}-\mathrm{cm}^{-3}$ curves and see that $q$ is expected to lie between $\sim 1$ and 4 at $1.55 \mathrm{eV}$. This range of $q$ can be compared with the range of $q$ that gives rise to the measured phase shift of $-58 \pm 5$. Using $q=-\tan (\phi)$, the measured phase shift corresponds to a range of $q$ between 1.3 and 2.0, which is entirely consistent with the range estimated from the Raman-scattering measurements.

Due to the simplicity of the Fano-Anderson Hamiltonian and the resulting classical equation of motions, it is worth considering potential limitations of the application to coherentphonon oscillations in $\mathrm{Si}$. The first potential limitation is the Fano-Anderson description of the background and its coupling to the discrete oscillator. As can be inferred from Eq. (1), in application to the Si problem, the background excitations describe valence-band electron-hole pairs. This description is more restrictive than the canonical practice of keeping explicit track of all electron states. ${ }^{33-35}$ However, because both the laser photons and the optic phonons have (essentially) zero momentum, momentum conservation restricts the coupled background to electron-hole pairs with zero total momentum, and so, the simpler Fano-Anderson background is not as restrictive as it might first appear. Second, in our model, the photons are introduced only at classical level. This approximation certainly cannot describe the complexity associated with a quantum description of the stimulated Raman processes that drive both the oscillator and the background. ${ }^{34}$ However, at $800 \mathrm{~nm}$, Raman excitation of the Si optic phonon is impulsive, ${ }^{30,31}$ and so, describing the photon excitation as a classical impulsive force may also not be unduly restrictive in this application. In order to assess these potential limitations of the present model, we are currently investigating a model for $\mathrm{Si}$ with a more standard description of the electronic excitations as well as a quantum description of the photons. ${ }^{36}$ Nonetheless, the results for the classical Fano oscillator do indicate that the discrete-continuum coupling should affect the phase of coherent-phonon oscillations in a system where Fano-like coupling occurs. Furthermore, the quantitative agreement between the model and the experimental results for Si suggests that the model presented here indeed captures the essence of this interaction in the time domain, at least, for this particular application.

\section{SUMMARY}

Starting with the Fano-Anderson Hamiltonian, ${ }^{19}$ we have studied the dynamics associated with classical coordinates derived from that Hamiltonian. When driven by external harmonic forces, the classical system exhibits the same Fano absorption line shape as the quantum-mechanical model when appropriate correspondences are made between the classical and the quantum parameters of the system. Thus, we take the equations of motion for the classical coordinates as the definition of the classical Fano oscillator. We have further studied the time dependence of the discrete-oscillator coordinate when the system is driven by forces that exist for a finite time. Under the condition that the forces are impulsive (and symmetric about $t=0$ ), the phase of the ensuing oscillations has a simple relationship to the Fano asymmetry parameter $q$ that appears in the expression for the absorption profile. 
These results indicate that the dynamics that give rise to the Fano line shape in the frequency domain also play a role in the time-domain response of coherently excited normal modes. We find good agreement between the predicted and the measured phases of the coherent oscillations of the zone-center optic phonon in degenerate $p$-type $\mathrm{Si}^{18}$

\section{ACKNOWLEDGMENTS}

The author would like the thank C. G. Torre for several enlightening conversations and a critical reading of the manuscript.

\section{APPENDIX A}

Here, we outline the derivation of the asymptotic result for the time dependence of the coordinate $Q(t)$, Eq. (30). To do this, we extend the technique used by Landau and Lifshitz to find the general solution of the undamped harmonic oscillator subject to a time-dependent force. ${ }^{37} \mathrm{We}$ start by introducing the functions,

$$
\begin{gathered}
\Psi^{(0)}=\frac{d Q^{(0)}}{d t}+i \Omega_{0} Q^{(0)}, \\
\psi_{k}^{(0)}=\frac{d q_{k}^{(0)}}{d t}+\left(\frac{\gamma_{k}}{2}+i \Omega_{k}\right) q_{k}^{(0)},
\end{gathered}
$$

and

$$
\Psi_{k}^{(1)}=\frac{d Q^{(1)}}{d t}+i \Omega_{0} Q_{k}^{(1)},
$$

and note that $Q^{(0)}=\operatorname{Im}\left(\Psi^{(0)}\right) / \Omega_{0}, q_{k}^{(0)}=\operatorname{Im}\left(\psi_{k}^{(0)}\right) / \Omega_{k}$, and $Q_{k}^{(1)}=\operatorname{Im}\left(\Psi_{k}^{(1)}\right) / \Omega_{0}$. This allows us to rewrite Eqs. (27)-(29) as the first-order equations,

$$
\begin{gathered}
\frac{d \Psi^{(0)}}{d t}-i \Omega_{0} \Psi^{(0)}=F_{Q}(t), \\
\frac{d \psi_{k}^{(0)}}{d t}-\left(i \Omega_{k}-\frac{\gamma_{k}}{2}\right) \psi_{k}^{(0)}=F_{k}(t),
\end{gathered}
$$

and

$$
\frac{d \Psi_{k}^{(1)}}{d t}-i \Omega_{0} \Psi_{k}^{(1)}=\frac{-\operatorname{Im}\left(\psi_{k}^{(0)} t\right)}{\Omega_{k}} .
$$

With the initial conditions given in Sec. III C, the solution to Eq. (A1) is

$$
\Psi^{(0)}(t)=\exp \left(i \Omega_{0} t\right) \int_{-\infty}^{t} d t^{\prime} F_{Q}\left(t^{\prime}\right) \exp \left(-i \Omega_{0} t^{\prime}\right) .
$$

In order to find the asymptotic limit of $\Psi^{(0)}(t)$, we consider Eq. (A6) for $t$ such that $F_{Q}(t)$ has vanished. In this case, we can replace the upper limit on the integral by $\infty$. If we define the Fourier transform of a time-dependent function $f(t)$ as

$$
\hat{f}(\Omega)=\frac{1}{\sqrt{2 \pi}} \int_{-\infty}^{\infty} d t f(t) \exp (i \Omega t)
$$

then we can simply express the asymptotic limit $\Psi^{(0)}(\bar{t})$ as

$$
\Psi^{(0)}(\bar{t})=\sqrt{2 \pi} \hat{F}_{Q}\left(-\Omega_{0}\right) \exp \left(i \Omega_{0} \bar{t}\right) .
$$

Similar to Eq. (A7), the solutions to Eqs. (A5) and (A6) may be written as

$$
\begin{aligned}
\psi_{k}^{(0)}(t)= & \exp \left[\left(i \Omega_{k}-\gamma_{k} / 2\right) t\right] \int_{-\infty}^{t} d t^{\prime} F_{k}\left(t^{\prime}\right) \\
& \times \exp \left[-\left(i \Omega_{k}-\gamma_{k} / 2\right) t^{\prime}\right],
\end{aligned}
$$

and

$$
\Psi_{k}^{(1)}(t)=\exp \left(i \Omega_{0} t\right) \int_{-\infty}^{t} d t^{\prime}\left[\frac{-\operatorname{Im}\left(\psi_{k}^{(0)}\left(t^{\prime}\right)\right)}{\Omega_{k}}\right] \exp \left(-i \Omega_{0} t^{\prime}\right) .
$$

The solution for $\Psi_{k}^{(1)}(t)$ is found by substituting the right-hand side of Eq. (A10) into Eq. (A11) for $\psi_{k}^{(0)}\left(t^{\prime}\right)$. Doing this and looking at the asymptotic limit $\Psi_{k}^{(1)}(\bar{t})$, it is straightforward to show that

$$
\Psi_{k}^{(1)}(\bar{t})=\sqrt{2 \pi} \frac{\hat{F}_{k}\left(-\Omega_{0}\right)}{\Omega_{0}^{2}-\omega_{k}^{2}-i \gamma_{k} \Omega_{0}} \exp \left(i \Omega_{0} \bar{t}\right) .
$$

Using the relations $Q^{(0)}=\operatorname{Im}\left(\Psi^{(0)}\right) / \Omega_{0}$ and $Q_{k}^{(1)}=$ $\operatorname{Im}\left(\Psi_{k}^{(1)}\right) / \Omega_{0}$, the results expressed by Eqs. (A9) and (A12) can be used in Eq. (25) to produce Eq. (30), the result for $Q(\bar{t})$.

\section{APPENDIX B}

Here, we look at the complete time dependence of $Q(t)$ for impulsive driving by Dirac $\delta$-function forces $F_{i}(t)=$ $\sqrt{2 \pi} \hat{F}_{i} \delta(t)$. [The constant $\hat{F}_{i}$ is the Fourier transform of $F_{i}(t)$.] In this case, the results in Appendix A can be used to easily obtain $Q^{(0)}(t)$ and $q_{k}^{(0)}(t)$ as

$$
Q^{(0)}(t)=\frac{\sqrt{2 \pi} \hat{F}_{Q}}{\Omega_{0}} \sin \left(\Omega_{0} t\right) \Theta(t),
$$

and

$$
q_{k}^{(0)}(t)=\frac{\sqrt{2 \pi} \hat{F}_{k}}{\Omega_{k}} \exp \left(-\gamma_{k} t / 2\right) \sin \left(\Omega_{k} t\right) \Theta(t) .
$$

Because $q_{k}^{(0)}=\operatorname{Im}\left(\psi_{k}^{(0)}\right) / \Omega_{k}$, this result for $q_{k}^{(0)}$ can be used in Eq. (A11) to find $\Psi_{k}^{(1)}(t)$ and thus $Q_{k}^{(1)}=\operatorname{Im}\left(\Psi_{k}^{(1)}\right) / \Omega_{0}$. Neglecting the nonresonant terms (which results in negligible error), the solution for $Q_{k}^{(1)}(t)$ can be written as

$$
\begin{aligned}
Q_{k}^{(1)}(t)= & \frac{\sqrt{2 \pi} \hat{F}_{k} \Theta(t)}{4 \Omega_{0} \Omega_{k}}\left\{\frac{\gamma_{k}\left[\cos \left(\Omega_{0} t\right)-\cos \left(\Omega_{k} t\right) \exp \left(-\gamma_{k} t / 2\right)\right]}{\left(\Omega_{k}-\Omega_{0}\right)^{2}+\gamma_{k}^{2} / 4}\right. \\
& \left.+\frac{2\left(\Omega_{k}-\Omega_{0}\right)\left[\sin \left(\Omega_{k} t\right) \exp \left(-\gamma_{k} t / 2\right)-\sin \left(\Omega_{0} t\right)\right]}{\left(\Omega_{k}-\Omega_{0}\right)^{2}+\gamma_{k}^{2} / 4}\right\} .
\end{aligned}
$$

As per Eq. (25), we now multiply Eq. (B3) by $c_{k}$ and sum over the continuum index $k$. In the limit $\gamma_{k}=\gamma \rightarrow 0$, the sums involving $\cos \left(\Omega_{0} t\right)$ and $\cos \left(\Omega_{k} t\right)$ equal zero, and we are left with

$$
\sum_{k} c_{k} Q_{k}^{(1)}(t)=\frac{\sqrt{2 \pi} \Theta(t)}{2 \Omega_{0}} \mathbb{P} \sum_{k} \frac{c_{k} \hat{F}_{k}}{\omega_{k}} \frac{\sin \left(\omega_{k} t\right)-\sin \left(\Omega_{0} t\right)}{\omega_{k}-\Omega_{0}} .
$$




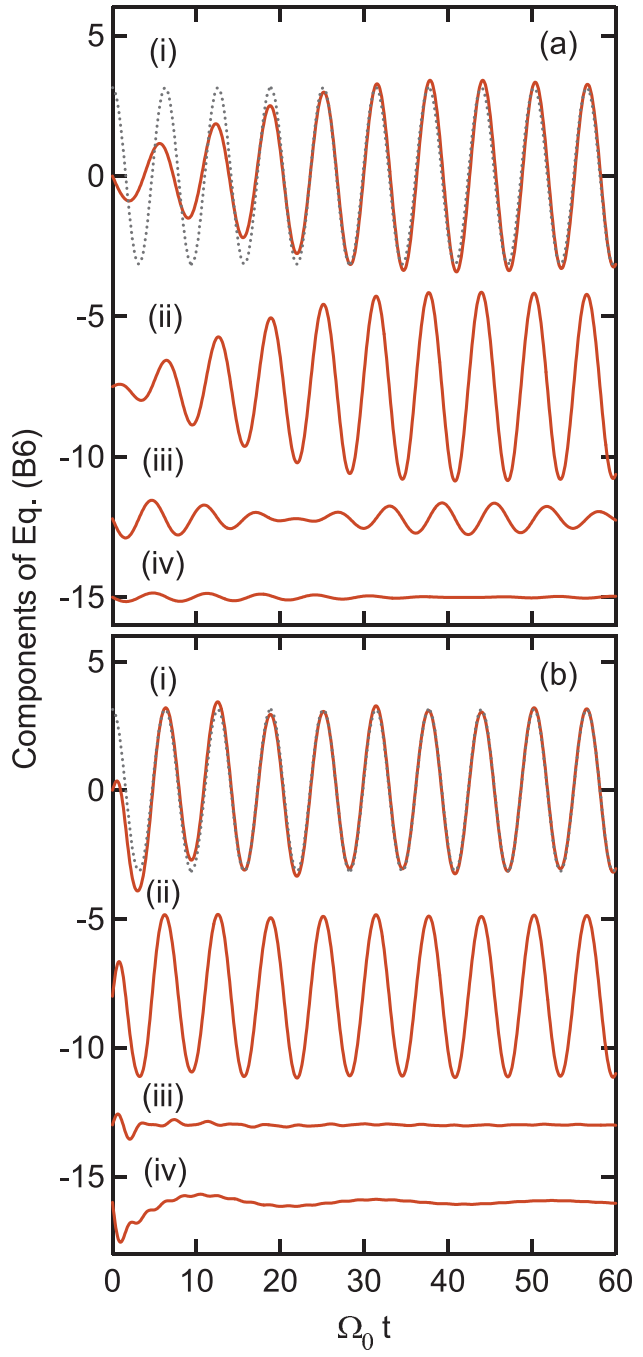

FIG. 4. (Color online) Components of Eq. (B6) vs $\Omega_{0} t$. Parts (a) and (b) correspond to narrow and wide continua, respectively (see text for details). The individual curves correspond to (i) the sum of terms inside the outermost parenthesis on the right-hand-side of Eq. (B6), (ii) the term containing $\cos \left(\Omega_{0} t\right)$, (iii) the term containing $\sin \left(\Omega_{0} t\right)$, and (iv) $\operatorname{Si}\left(\omega_{\min } t\right)-\operatorname{Si}\left(\omega_{\max } t\right)$. Curves (ii)-(iv) are offset for clarity. The asymptotic limit $\pi \cos \left(\Omega_{0} t\right)$ is plotted (dotted curve) with (i).

Combining this result with Eq. (B1), we have the final result for $Q(t)$ with $\delta$-function driving,

$$
\begin{aligned}
Q(t)= & \frac{\sqrt{2 \pi} \Theta(t)}{\Omega_{0}}\left[\left(\hat{F}_{Q}+\mathbb{P} \sum_{k} \frac{c_{k} \hat{F}_{k}}{2 \omega_{k}\left(\Omega_{0}-\omega_{k}\right)}\right) \sin \left(\Omega_{0} t\right)\right. \\
& \left.+\mathbb{P} \sum_{k} \frac{c_{k} \hat{F}_{k} \sin \left(\omega_{k} t\right)}{2 \omega_{k}\left(\Omega_{0}-\omega_{k}\right)}\right] .
\end{aligned}
$$

We first note that the term containing $\sin \left(\Omega_{0} t\right)$ in Eq. (B5) is identical to the likewise term in Eq. (32). Thus, for a $\delta$-function impulse, this part of the asymptotic result is valid for all $t>0$. The remaining term in Eq. (B5) evolves into the $\cos \left(\Omega_{0} t\right)$ term in Eq. (32).

To investigate the evolution of this term, we consider a simplified case where $c_{k}$ and $\hat{F}_{k}$ vary slowly enough that they may be taken as independent of $k$, i.e., $c_{k}=c$ and $\hat{F}_{k}=\hat{F}_{B}$. We further assume that the background density of states $g\left(\omega_{k}\right)$ is constant $\left.\left[g\left(\omega_{k}\right)=g\right)\right]$ over a range of frequencies from $\omega_{\min }$ to $\omega_{\max }$ and is zero elsewhere. With these assumptions, the last term in Eq. (B5) can be replaced by an integral over the continuum frequencies. Evaluating the integral, we obtain

$$
\begin{aligned}
\mathbb{P} \sum_{k} \frac{c_{k} \hat{F}_{k} \sin \left(\omega_{k} t\right)}{2 \omega_{k}\left(\Omega_{0}-\omega_{k}\right)} \\
=\frac{c g \hat{F}_{B}}{2 \Omega_{0}}\left(\left\{\operatorname{Si}\left[\left(\omega_{\max }-\Omega_{0}\right) t\right]+\operatorname{Si}\left[\left(\Omega_{0}-\omega_{\min }\right) t\right]\right\} \cos \left(\Omega_{0} t\right)\right. \\
\quad+\left\{\operatorname{Ci}\left[\left(\omega_{\max }-\Omega_{0}\right) t\right]-\operatorname{Ci}\left[\left(\Omega_{0}-\omega_{\min }\right) t\right]\right\} \sin \left(\Omega_{0} t\right) \\
\left.\quad+\operatorname{Si}\left(\omega_{\min } t\right)-\operatorname{Si}\left(\omega_{\max } t\right)\right),
\end{aligned}
$$

where $\operatorname{Si}(x)$ and $\operatorname{Ci}(x)$ are the sine-integral and cosineintegral functions, respectively. Before considering the time dependence of Eq. (B6) in detail, we note that, because $\mathrm{Si}(x) \rightarrow \pi / 2$ and $\mathrm{Ci}(x) \rightarrow 0$ for large $x$, the asymptotic limit of Eq. (B6) is given by

$$
\mathbb{P} \sum_{k} \frac{c_{k} \hat{F}_{k} \sin \left(\omega_{k} \bar{t}\right)}{2 \omega_{k}\left(\Omega_{0}-\omega_{k}\right)}=\frac{\pi c g \hat{F}_{B}}{2 \Omega_{0}} \cos \left(\Omega_{0} \bar{t}\right)
$$

We further note that, under the assumptions applicable to Eq. (B7), the term containing $\cos \left(\Omega_{0} t\right)$ in Eq. (31) becomes identical to this last expression.

In Fig. 4, we plot the functions contained in the outermost parenthesis on the right-hand side of Eq. (B6) for two example continua. Figure 4(a) is for a fairly narrow continuum: $\omega_{\min } / \Omega_{0}=0.9$ and $\omega_{\max } / \Omega_{0}=1.05$, while Fig. 4(b) is for a much wider continuum: $\omega_{\min } / \Omega_{0}=0.3$ and $\omega_{\max } / \Omega_{0}=3.0$. As can be ascertained from the figure, in both cases, the contributions from the $\left\{\operatorname{Ci}\left[\left(\omega_{\max }-\Omega_{0}\right) t\right]-\operatorname{Ci}\left[\left(\Omega_{0}-\omega_{\min }\right) t\right]\right\} \sin \left(\Omega_{0} t\right)$ and $\left[\operatorname{Si}\left(\omega_{\min } t\right)-\operatorname{Si}\left(\omega_{\max } t\right)\right]$ terms are relatively insignificant. Furthermore, because $\operatorname{Si}(x)$ becomes nearly equal to its asymptotic limit for $x \approx 2$, the term containing $\cos \left(\Omega_{0} t\right)$ becomes nearly equal to its asymptotic limit on a time scale given by the larger of $2 /\left(\omega_{\max }-\Omega_{0}\right)$ and $2 /\left(\Omega_{0}-\right.$ $\left.\omega_{\min }\right)$.

These results indicate that time-domain data may also provide information on the bandwidth of the coupled continuum. For example, the coherent-phonon data from degenerate $p$-type $\mathrm{Si}$ are well fit by a single oscillator with constant phase, ${ }^{18}$ indicating that the asymptotic limit is established very quickly, as illustrated by Fig. 4(b). This observation suggests that the bandwidth of the coupled continuum is fairly wide for degenerate $p$-type $\mathrm{Si}$. Indeed, for $p$-type $\mathrm{Si}$ with a hole concentration on the order of $10^{20} \mathrm{~cm}^{-3}$, the coupled bandwidth is significantly larger that the opticphonon energy of $0.064 \mathrm{eV}^{7}$ In contrast, high-laser-intensity coherent-phonon data from $\mathrm{Zn}$ exhibit a significant phase difference between early and late times, ${ }^{16}$ indicating that it takes a few cycles to reach the asymptotic limit, as is the case for the narrow-bandwidth results shown in Fig. 4(a). 
${ }^{1}$ U. Fano, Phys. Rev. 124, 1866 (1961).

${ }^{2}$ J. C. Phillips, Phys. Rev. 136, A1705 (1964).

${ }^{3}$ Y. J. Chabal, Phys. Rev. Lett. 55, 845 (1985).

${ }^{4}$ D. C. Langreth, Phys. Rev. Lett. 54, 126 (1985).

${ }^{5}$ R. S. Sorbello, Phys. Rev. B 32, 6294 (1985).

${ }^{6}$ F. Cerdeira, T. A. Fjeldly, and M. Cardona, Solid State Commun. 13, 325 (1973).

${ }^{7}$ F. Cerdeira, T. A. Fjeldly, and M. Cardona, Phys. Rev. B 8, 4734 (1973).

${ }^{8}$ B. G. Burke, J. Chan, K. A. Williams, Z. Wu, A. A. Puretzky, and

D. B. Geohegan, J. Raman Spectrosc. 41, 1759 (2010).

${ }^{9}$ B. Luk'yanchuk, N. I. Zheludev, S. A. Maier, N. J. Halas, P. Nordlander, H. Giessen, and C. T. Chong, Nature Mater. 9, 707 (2010).

${ }^{10}$ M. Balkanski, K. P. Jain, R. Beserman, and M. Jouanne, Phys. Rev. B 12, 4328 (1975).

${ }^{11}$ V. I. Belitsky, A. Cantarero, M. Cardona, C. Trallero-Giner, and S. T. Pavlov, J. Phys.: Condens. Matter 9, 5965 (1997).

${ }^{12}$ F. Cerdeira, T. A. Fjeldly, and M. Cardona, Phys. Rev. B 9, 4344 (1974).

${ }^{13}$ M. Chandrasekhar, H. R. Chandrasekhar, M. Grimsditch, and M. Cardona, Phys. Rev. B 22, 4825 (1980).

${ }^{14}$ M. Hase, M. Kitajima, A. M. Constantinescu, and H. Petek, Nature (London) 426, 51 (2003).

${ }^{15}$ J. D. Lee, J. Inoue, and M. Hase, Phys. Rev. Lett. 97, 157405 (2006).

${ }^{16}$ M. Hase, J. Demsar, and M. Kitajima, Phys. Rev. B 74, 212301 (2006).

${ }^{17}$ O. V. Misochko, K. Ishioka, M. Hase, and M. Kitajima, J. Phys.: Condens. Matter 19, 156227 (2007).

${ }^{18}$ K. Kato, A. Ishizawa, K. Oguri, K. Tateno, T. Tawara, H. Gotoh, M. Kitajima, and H. Nakano, Jpn. J. Appl. Phys. 48, 100205 (2009).

${ }^{19}$ G. D. Mahan, Many-Particle Physics (Plenum, New York, 1981).

${ }^{20}$ A. Shibatani and Y. Toyozawa, J. Phys. Soc. Jpn. 25, 335 (1968).

${ }^{21}$ M. W. Klein, T. Tritschler, M. Wegener, and S. Linden, Phys. Rev. B 72, 115113 (2005).
${ }^{22}$ Y. S. Joe, A. M. Satanin, and C. S. Kim, Phys. Scr. 74, 259 (2006).

${ }^{23}$ A. S. Barker and J. J. Hopfield, Phys. Rev. 135, A1732 (1964).

${ }^{24}$ V. V. Struzhkin, A. F. Goncharov, R. J. Hemley, and H.-k. Mao, Phys. Rev. Lett. 78, 4446 (1997).

${ }^{25}$ S. Mukherjee, H. Sobhani, J. B. Lassiter, R. Bardhan, P. Nordlander, and N. J. Halas, Nano Lett. 10, 2694 (2010).

${ }^{26}$ N. A. Mirin, K. Bao, and P. Nordlander, J. Phys. Chem. A 113, 4028 (2009).

${ }^{27}$ K. Bao, H. Sobhani, and P. Nordlander, Chin. Sci. Bull. 55, 2629 (2010).

${ }^{28}$ From a practical point of view, any two-phase values that differ by $\pm \pi$ are equivalent as this difference only corresponds to an overall sign change in $Q(t)$.

${ }^{29}$ A. Compaan and H. J. Trodahl, Phys. Rev. B 29, 793 (1984).

${ }^{30}$ D. M. Riffe and A. J. Sabbah, Phys. Rev. B 76, 085207 (2007).

${ }^{31}$ Y. Shinohara, K. Yabana, Y. Kawashita, J. I. Iwata, T. Otobe, and G. F. Bertsch, Phys. Rev. B 82, 155110 (2010).

${ }^{32}$ Kato et al. actually report a smaller phase for the degenerate sample as compared to the undoped sample. However, by plotting $\cos \left(\Omega_{0} t+\phi\right)$ with their reported values of $\phi$, it is clear from their Fig. 2 that the signs of their reported phases are incorrect. They also report the phase of the undoped sample as being significantly different than $\pm 90^{\circ}$, which does not agree with previously reported measurements on nondegenerate Si (Ref. 30) and time-dependent density-functional theory. ${ }^{31}$ We obtain the value of $-58 \pm 5^{\circ}$ for the degenerate sample by using the difference of $32 \pm 5^{\circ}$ found in the paper by Kato $e t a l$. along with a value of $-90^{\circ}$ for nondegenerate Si.

${ }^{33}$ A. V. Kuznetsov and C. J. Stanton, Phys. Rev. Lett. 73, 3243 (1994).

${ }^{34}$ R. Merlin, Solid State Commun. 102, 207 (1997).

${ }^{35}$ F. T. Vasko and O. E. Raichev, Quantum Kinetic Theory and Applications (Springer, New York, 2005).

${ }^{36}$ D. M. Riffe and C. G. Torre (unpublished).

${ }^{37}$ L. D. Landau and E. M. Lifshitz, Mechanics, 3rd ed. (Pergamon, New York, 1976). 